\title{
Young addicted men hormone profile detection
}

\author{
Paweł Zieliński ${ }^{a, b}$, Piotr Wąsiewicz ${ }^{a}$, Bożena Leszczyńska ${ }^{c}$, Joanna Gromadzka-Ostrowska $^{c}$ \\ ${ }^{a}$ Institute of Electronic Systems, Warsaw University of Technology \\ ${ }^{b}$ Interdisciplinary Centre for Mathematical and Computational Modeling, University of Warsaw \\ ${ }^{c}$ Department of Dietetic, Warsaw University of Life Sciences
}

\begin{abstract}
Hormone parameters were determined in the serum of young addicted men in order to compare them with those obtained from the group of healthy subjects. Three groups were investigated which were named opiates, mixed and control group. Statistical and data mining methods were applied to obtain significant differences. R package was used for all computation. The determination of hormones parameters provide important information relative to impact of addiction.
\end{abstract}

Keywords: opiate addicts, hormone profile, cortisol, testosterone, insulin, leptin, growth hormone

\section{INTRODUCTION}

Addiction is a serious social problem and has destructive influence on health and quality of living of an inflicted person. Determinants of addictive disease may be such factors as genetic predisposition, brain reward mechanisms, socio-cultural context and psychological profile. Consequences of drug dependence are social, biological and psychological, and almost always interact. Addiction is large problem for society, cost of health care and social implication are difficult to measure. Highly addictive drugs are noticed ${ }^{1}$ to decrease cell growth and division. They are also well known to be associated with physical disorders. The hormone system of drug dependent individuals is dysfunctional and hyper-stimulated.

Opioids such as morphine or methadone are also useful. The analgesic efficacy of opioids in neuropathic pain is undoubted. But in recent clinical studies prolonged opioids treatment ${ }^{2,3}$ were reported to cause a strong hormonal and immune changes. So one of fundamental principles of pain management is appropriate dosing without side effects, because hormonal effects are most likely to occur in patients who receive high doses of opioids in long period of time.

The differences observed in other metabolic biomarkers are also interest. Considerable research and community attention was made. Chemical addiction is shown to accelerate ageing ${ }^{1}$ and have developmental implications. ${ }^{4}$ Pharmacogenomics research ${ }^{5}$ showed that opiates addiction is in large degree genetically transmitted and genetic risk factors are separated from other drugs of abuse. Neurochemical mechanisms of opioid reward was also investigated. ${ }^{6}$ Other study $^{7}$ showed effect of sport activity reversing hyperinsulinemia in the ex-addicts. Numerous studies ${ }^{8-10}$ are based on animal models since they well imitate addiction in humans. Some investigations ${ }^{11}$ inspired by studies on rodents revealed unknown effects in humans. In Ref. 12 depression was associated with high cortisol concentration, but this association was evident in women. People's beliefs were also investigated ${ }^{13}$ and the addict is seen as a victim of society. Although such progress further investigations are needed to achieve deeper understanding of the underlying mechanisms of addiction.

Consideration of addiction impact on hormone profile is the scope of the present investigation. The association between hormones and addiction was determined. Hormone profile was also made.

Further author information: (Send correspondence to Paweł Zieliński)

Paweł Zieliński: E-mail: pzielins@mion.elka.pw.edu.pl

Piotr Wąsiewicz: E-mail: pwasiewi@elka.pw.edu.pl

Bożena Leszczyńska: E-mail: bozena_leszczynska@interia.pl>

Joanna Gromadzka-Ostrowska: E-mail: joanna_gromadzka_ostrowska@sggw.pl

Photonics Applications in Astronomy, Communications, Industry, and High-Energy Physics Experiments 2010,

edited by Ryszard S. Romaniuk, Krzysztof S. Kulpa, Proc. of SPIE Vol. 7745, 77450V

(C) 2010 SPIE · CCC code: 0277-786X/10/\$18 - doi: 10.1117/12.872080

Proc. of SPIE Vol. 7745 77450V-1 


\section{MEDICAL MEASUREMENTS}

The study was performed among 119 patients with a history of drug or alcohol abuse and 36 healthy volunteers recruited from students. Inclusion criteria were the following: only young men were investigated (mean age \pm standard deviation: $21.7 \pm 3.2$ years), clinically stable. Raw data were collected by medical equipment. Drug or alcohol abuse history and category of addiction were self-reported by validated questionnaires. Nutrition consumption was monitored by 24-hours dietary intake common questionnaire. List of food, snacks and drink was included. For the dietary habits assessment self-reported qualitative food frequency questionnaire was used.All studies were consistent with good clinical practice. ${ }^{14}$

\section{DATA RETRIEVAL METHODOLOGY}

Statistical analysis and data-mining were performed using $\mathrm{R}$ framework. ${ }^{15}$ Some data, such outliers, was excluded from the analysis. The Kruskal-Wallis rank sum ${ }^{16}$ test was used to compare the mean differences between measured hormones levels. It is a non-parametric test used when normality assumptions aren't satisfied and the analysis of variance can't be applied.

After statistic methods data-ming technics were applied. We used dendrograms to obtain number of means and clustering ${ }^{16}$ to investigate hormone profile. In general, a grouping is method to show similar objects near in features space. This space can be visualized in two dimensions after scaling based e.g. on principle component analysis (PCA), which transform real dimensions to artificial ones, where first ones have the most correlations within and the rest of dimensions may be omitted with small resulting errors.

\section{DATA RETRIEVAL RESULTS}

During measurements sets were obtained from three groups, which were named a control (healthy volunteers) and two test groups (opiates and mixed). The test group mixed was consisted of 66 patients, the test group opiates contain 53 patients and the test one of healthy young student staff 36 people. In some calculation some data were removed due to missing values of hormone levels.

\subsection{Statistical analysis}

Each hormone level was measured and statistical methods were applied. We visualized our result to show differences in groups. Density plot and box-whisker plot were used to show distribution of measured variables.

\subsubsection{Cortisol}

The current result (Fig. 1) showed significant differences in mean values (Kruskal-Wallis chi-squared $=8.9, \mathrm{df}=2, \mathrm{p}=$ 0.01). Reason for this result may be detoxification which is responses for hypercortisolism in males addicted to heroin and stress-like response for alcohol and nicotine in mixed group. Other possible explanation for this effect could be experiencing withdrawal symptoms in both addicted groups. Cortisol is quite responsive for psychological stress ${ }^{17}$ which suggested that increased level of cortisol may by result of stressful environment. These possibilities remain to be examined, because depend of addiction type high level of cortisol may have different pathways. ${ }^{17}$

\subsubsection{Growth hormone}

Small difference in growth hormone (Fig. 2) was observed between groups, but our research did not reach significance (Kruskal-Wallis chi-squared $=0.3961, \mathrm{df}=2, \mathrm{p}=0.82$ ). Larger studies would have more statistical power to detect such alterations, because recent studies made by Reece ${ }^{1}$ showed difference in IGF-1 protein with production is stimulated by growth hormone.

\subsubsection{Insulin}

Significant differences (Fig. 3) in insulin levels Kruskal-Wallis chi-squared $=28.4$, df $=2, p<0.001$ was obtained. It is difficult to assume whether the pharmacology of addiction itself, or poor nutrition or overdose is largely responsible for the observed effects. It is also known that during abstention drug abusers have eating disorders. ${ }^{7}$ 

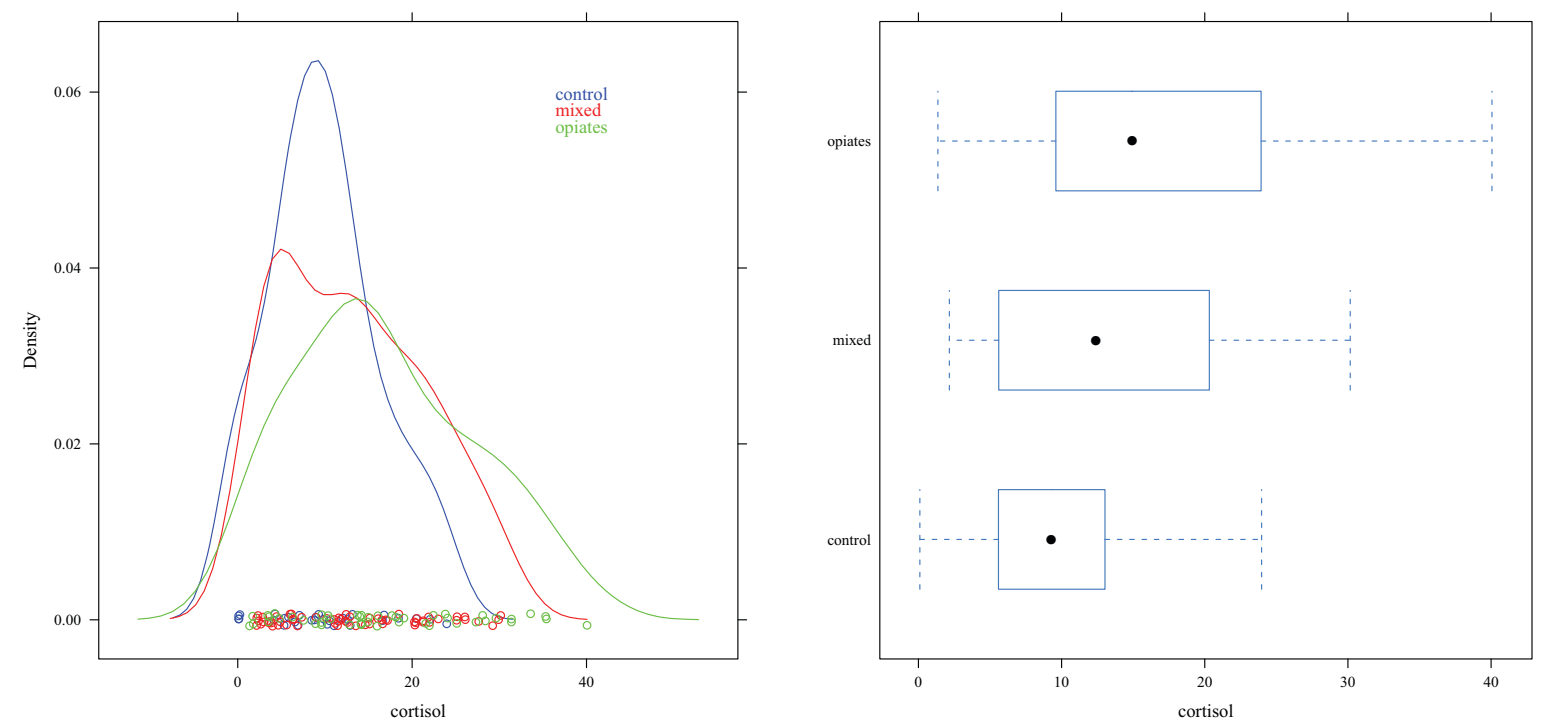

Figure 1. Cortisol density plot and box plot.
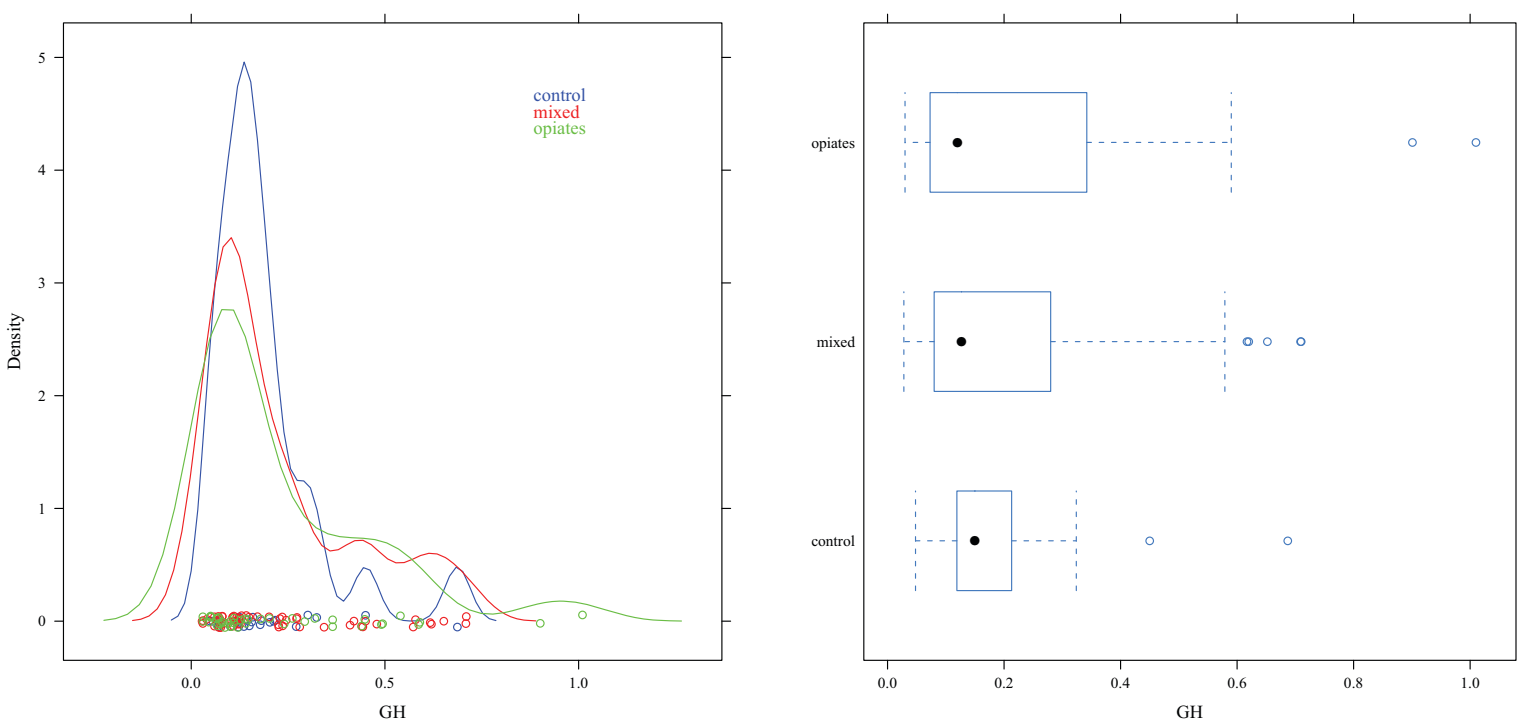

Figure 2. Growth hormone density plot and box plot. 

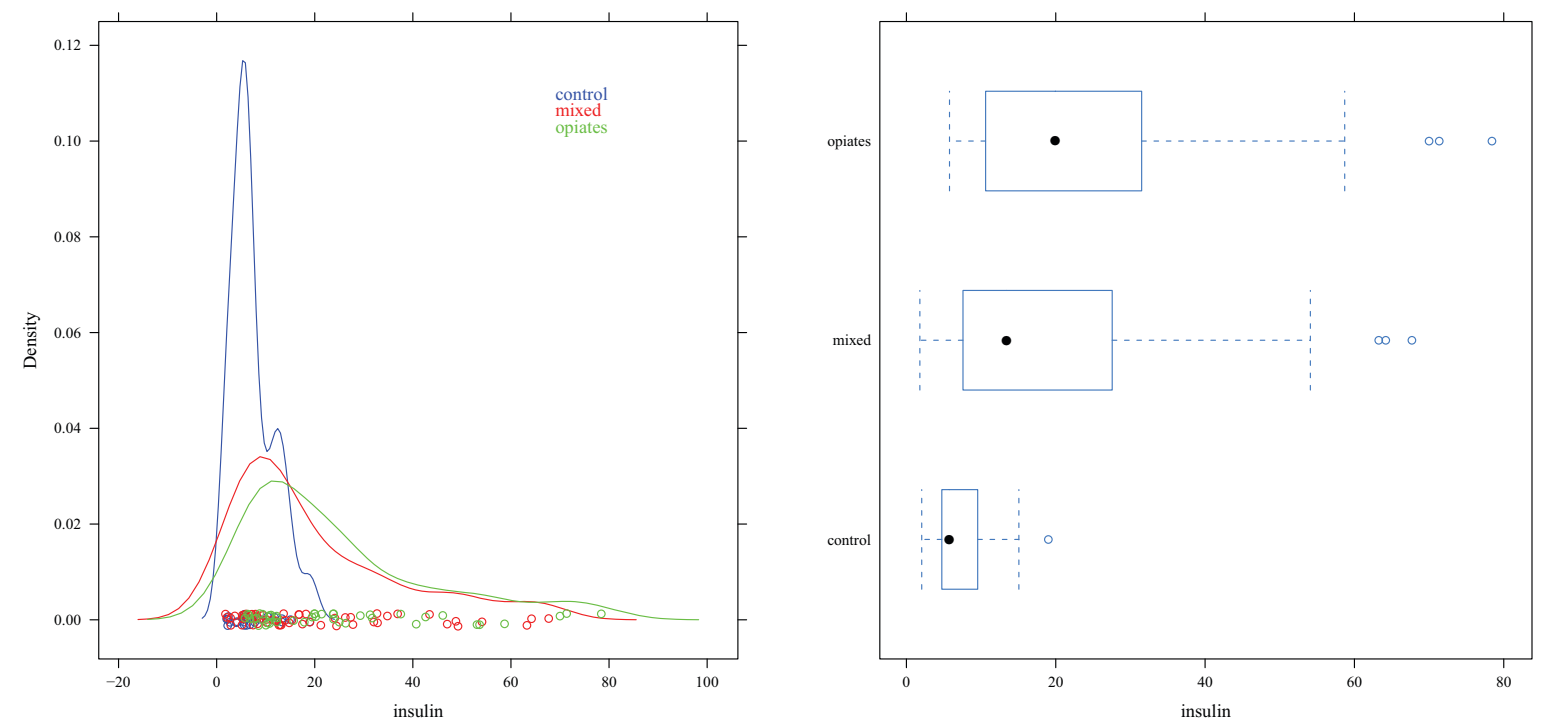

Figure 3. Insulin density plot and box plot.
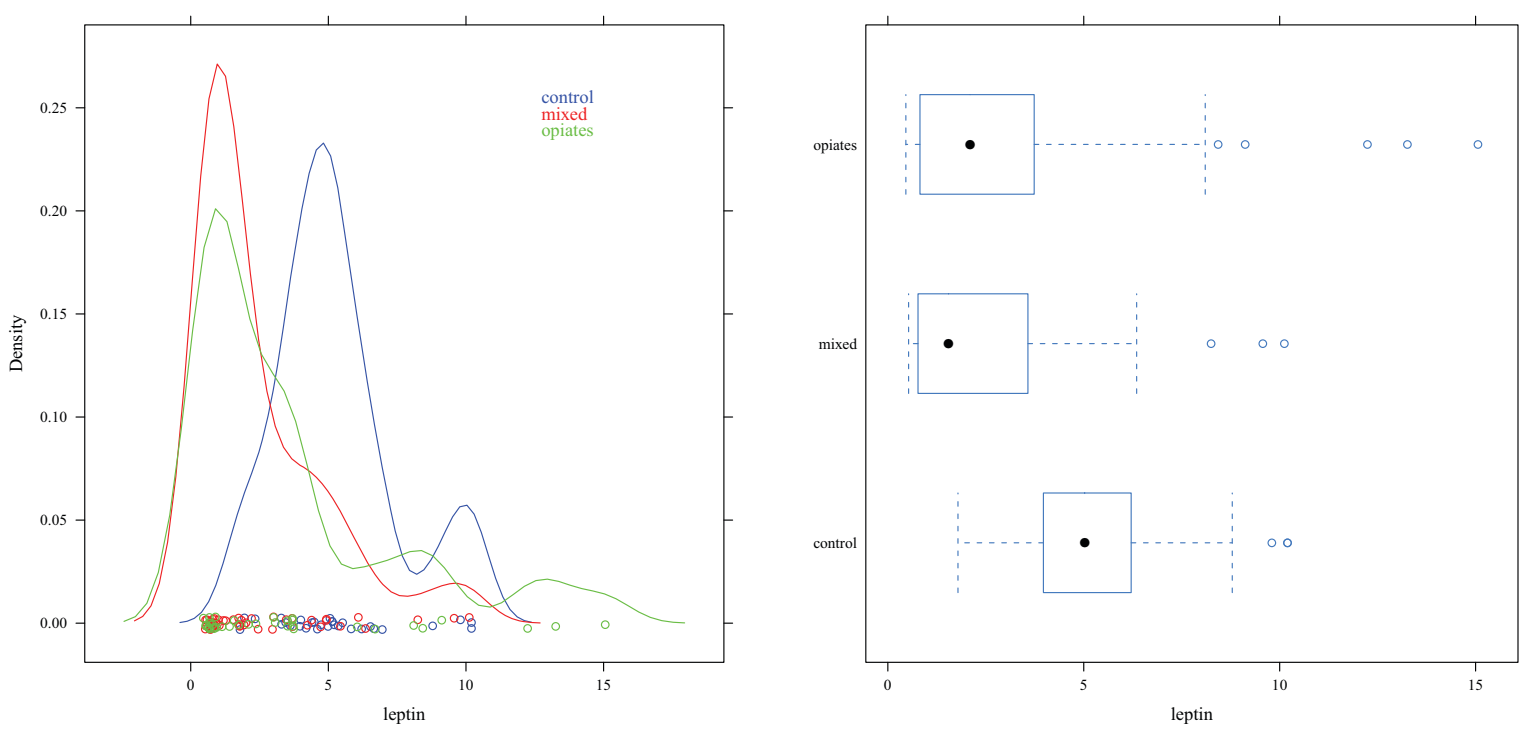

Figure 4. Leptin density plot and box plot. 

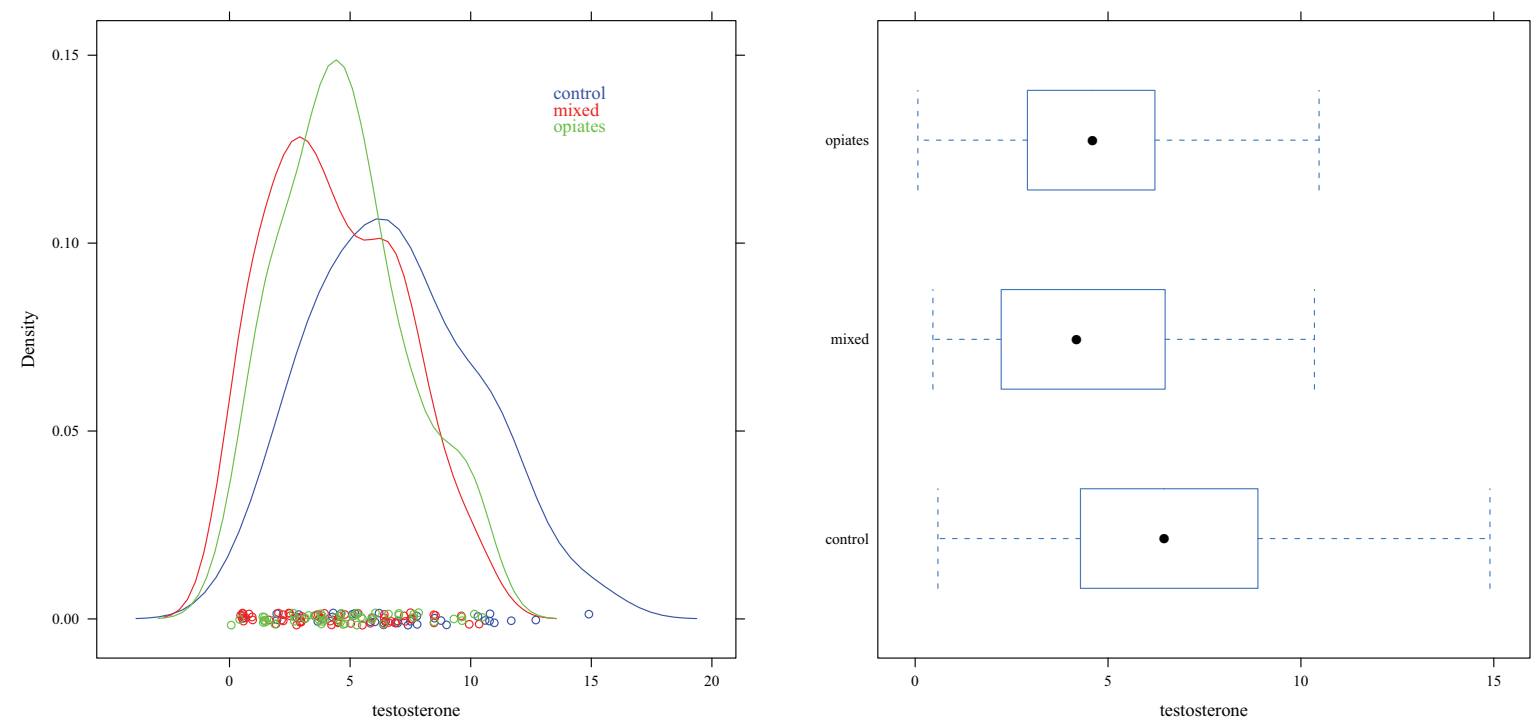

Figure 5. Testosterone density plot and box plot.

\subsubsection{Leptin}

Insulin levels (Fig. 4) also differ significantly (Kruskal-Wallis chi-squared $=25.2, \mathrm{df}=2, \mathrm{p}<0.001$ ). Decreasing leptin association with abstinence duration was reported ${ }^{18,19}$ what may have impact on mixed mean leptin value.

\subsubsection{Testosterone}

This finding (Fig. 5) is consistent with the existing literature in that opiates decrease level of testosterone (Kruskal-Wallis chi-squared $=12.9, \mathrm{df}=2, \mathrm{p}=0.001$ ).

\subsection{Data mining}

\subsubsection{Hormone profile}

In Fig. 6 the all hormone variable cluster dendrogram is depicted. The shorter arms the more similar hormone profile of patients.

Based on PCA k-means clustering performed on measured hormone levels revealed that artificial clusters are connected with addiction groups. It can be clearly seen because colors of figures are connected with addiction group. We applied color after unsupervised learning. First group (circles Fig. 6) is dominated by mixed and opiates individuals. Second group (triangles) contains mixed addiction persons and only one person from control group which is in great distance from them. Third group (crosses) is composed of control group and few individuals from other two. Fourth group can be omitted because is very small. Fifth group (rhombus) contains mixed and opiates individuals and is also small like previous one.

\section{SUMMARY}

The presented results has improved our understanding of how addiction acts on hormone profile. Unsupervised method such as clustering is adequate in the case of hormone parameters and detect interesting groups in this data set. Statistical methods and graphics visualization has shown implication of addiction on measured hormones levels.

Further clinical based investigation of the dynamic interaction between addiction and hormones levels can improve clinical management of addicted part of population and opiates based treatment in chronic pain. 

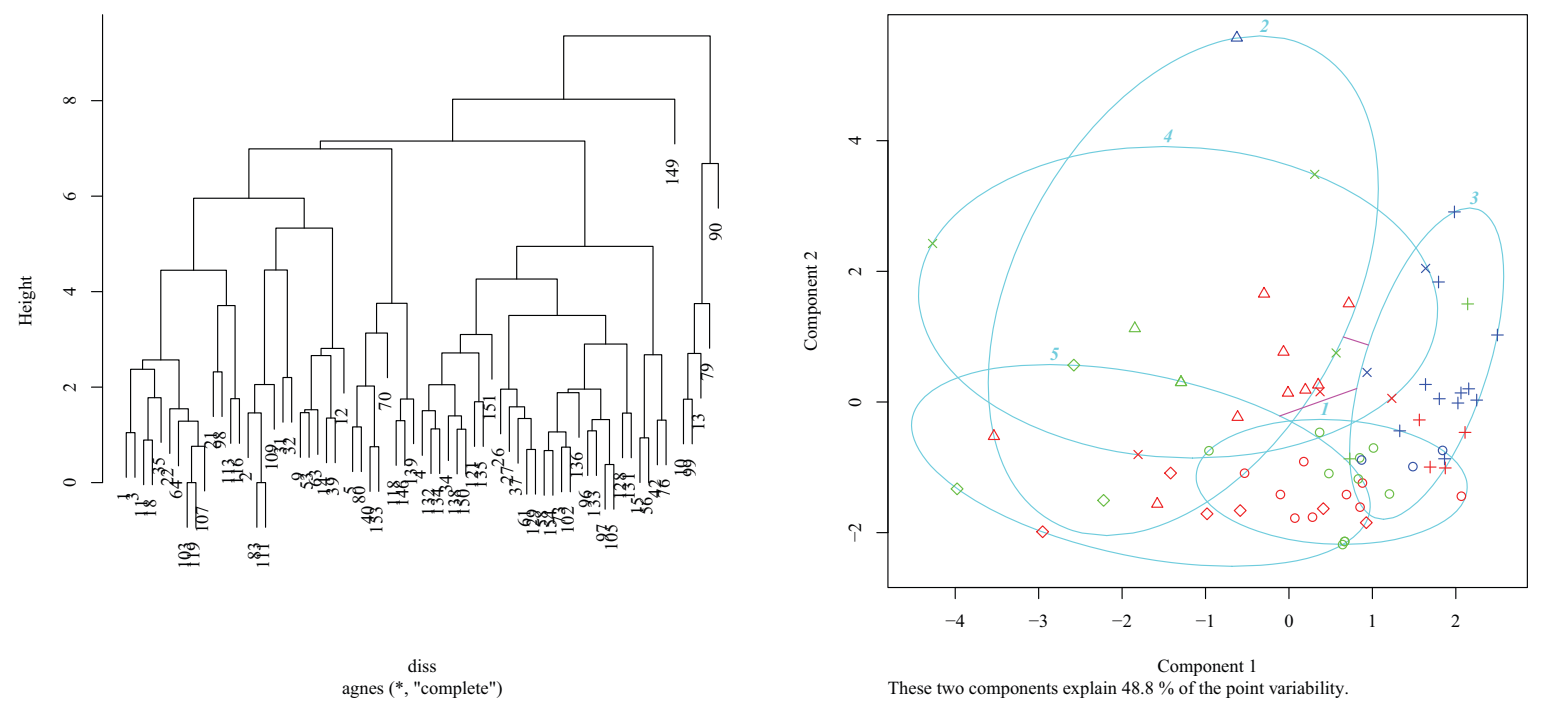

Figure 6. Results obtained for clustering.

\section{REFERENCES}

[1] Reece, A. S., "Evidence of accelerated ageing in clinical drug addiction from immune, hepatic and metabolic biomarkers," Immunity and Ageing 4 (Sept. 2007).

[2] Ballantyne, J. C. and Mao, J., "Opioid therapy for chronic pain," New England Journal of Medicine 349, 1943-1953 (Nov. 2003).

[3] Højsted, J. and Sjøgren, P., "Addiction to opioids in chronic pain patients: A literature review," European Journal of Pain 11, 490-518 (July 2007).

[4] Mello, N. K., "Hormones, nicotine, and cocaine: Clinical studies," Hormones and Behavior 58, 57-71 (June 2010).

[5] Lichtermann, D., Franke, P., Maier, W., and Rao, M. L., "Pharmacogenomics and addiction to opiates," European Journal of Pharmacology 27, 269-279 (Dec. 2000).

[6] Przewlocki, R., "Opioid abuse and brain gene expression," European Journal of Pharmacology 500, 331-349 (Oct. 2004).

[7] Hunt DD, Hou CW, Lai YC, Lin FC, Chen CY, Lin CH, Liao YH, Lee WC, Chen JJ, and Kuo CH, "Effects of hiking at altitude on body composition and insulin sensitivity in recovering drug addicts.," Preventive Medecine 39, 681-688 (Oct. 2004).

[8] Anton, B. and Leff, P., "A novel bivalent morphine/heroin vaccine that prevents relapse to heroin addictionnext term in previous termrodentsnext term," Vaccine 24, 3232-3240 (Apr. 2006).

[9] Grupp, L. A., Hsu, G., Ng, N., and Harding, S., "Glucose and the insulin-releasing drug tolbutamide attenuate the effects of morphine and angiotensin on alcohol consumption," Alcohol 14, 71-79 (Jan. 1997).

[10] Faraday, M. M., Blakeman, K. H., and Grunberg, N. E., "Strain and sex alter effects of stress and nicotine on feeding, body weight, and hpa axis hormones," Pharmacology Biochemistry and Behavior 80, 577-589 (Jan. 2005).

[11] Elman, I. and Lukas, S. E., "Effects of cortisol and cocaine on plasma prolactin and growth hormone levels in cocaine-dependent volunteers," Addictive Behaviors 30, 859-864 (Sept. 2005).

[12] Wisniewski, A. B., Brown, T. T., John, M., Cofranceso Jr., J., Golub, E. T., Ricketts, E. P., Wand, G., and Dobs, A. S., "Cortisol levels and depression in men and women using heroin and cocaine," Psychoneuroendocrinology 30, 250-255 (Feb. 2006).

[13] Furnham, A. and Thomson, L., "Lay theories of heroin addiction," Social Science and Medicine 43, 29-40 (Nov. 1996). 
[14] Walker, R. D., Howard, M. O., Walker, P. S., Lambert, M. D., and Suchinsky, R., "Practice guidelines in the addictions: Recent developments," Journal of Substance Abuse Treatment 12, 63-73 (Mar. 1995).

[15] R Development Core Team, R: A Language and Environment for StatisticalComputing. R Foundation for Statistical Computing, Vienna, Austria (2010). ISBN 3-900051-07-0.

[16] Venables, W. N. and Ripley, B. D., [Modern applied statistics with S], Springer-Verlag, New York, 4th ed. (2002).

[17] Lovallo, W. R., "Cortisol secretion patterns in addiction and addiction risk," International Journal of Psychophysiology 59, 195-202 (Mar. 2006).

[18] Kiefer, F., Jahn, H., Otte, C., Demiralay, C., Wolf, K., and Wiedemann, K., "Increasing leptin precedes craving and relapse during pharmacological abstinence maintenance treatment of alcoholism," Journal of Psychiatric Research 39, 545-551 (Sept. 2005).

[19] Hillemacher, T., Bleich, S., Frielinga, H., Schanzea, A., Wilhelma, J., Sperlinga, W., Kornhubera, J., and Kraus, T., "Evidence of an association of leptin serum levels and craving in alcohol dependence," Psychoneuroendocrinology 32, 87-90 (Jan. 2007). 